\title{
PARÁMETROS DE DISEÑO Y DESEMPEÑO ENERGÉTICO EN EDIFICIOS DE CLIMA MEDITERRÁNEO ${ }^{1}$
}

\author{
FELIPE ENCINAS - WALDO BUSTAMANTE
}

Contrariamente a lo que puede aparecer a simple vista, el análisis de sensibilidad -como herramienta más propia de un ejercicio de ingeniería-puede también entenderse como un elemento esencial e inherente a la práctica arquitectónica. En efecto, lo que este tipo de análisis matemático hace, es cambiar sistemáticamente los parámetros de entrada en un modelo para determinar los efectos que ocurren a partir de dichas modificaciones.

Las técnicas de representación 2D y 3D utilizadas habitualmente en arquitectura constituyen el output o salida tradicional de estas modificaciones en cuanto a aspectos formales; vale decir, ya sea mediante planimetría, maqueta o visualización digital 3D, es posible dar cuenta de cómo las variaciones en los parámetros de entrada determinan la forma. Pero, ¿qué es lo que pasa si además de evaluar cómo se ve afectada la forma arquitectónica, interesa medir cuantitativamente el impacto de estas modificaciones en el desempeño energético ${ }^{2}$ del proyecto?

No se descubrirá ahora la importancia de los aspectos de desempeño en la discusión arquitectónica contemporánea. Ya sea debido a legítimas preocupaciones ambientales, consideraciones con respecto a la economía de recursos o sobre el confort y bienestar de los usuarios de los espacios que se proyectan, se puede convenir en la relevancia de prever el impacto que las decisiones de diseño tendrán sobre el desempeño energético del proyecto, una vez construido y habitado.

A muchos arquitectos no les resultaría extraño el supuesto de que, en un clima mediterráneo como el de Santiago de Chile, la orientación sea una variable altamente significativa en relación a la demanda de enfriamiento en un edificio. Sin embargo, como se verá más adelante a la luz de los resultados, la orientación podría constituir la última preocupación dentro de las diferentes decisiones de diseño a las que un arquitecto se puede enfrentar al proyectar un edificio desde la perspectiva de su desempeño energético en refrigeración.

PARÁMETROS DE DISEÑO

Claramente las simulaciones digitales de comportamiento térmico han contribuido a despejar las dudas acerca de cómo los distintos parámetros de diseño arquitectónico afectan el desempeño energético de un proyecto. Entre estos parámetros se podrían mencionar justamente la orientación y el tipo de vidrio, pero también aspectos como la materialidad de la envolvente opaca o incluso consideraciones relativas a la forma, como volumen, organización de la planta y otros. Luego, al variar una por una las especificaciones de cada parámetro -por ejemplo, el espesor de aislante térmico de los muros-, es posible obtener algunas conclusiones acerca de cómo cada una de estas modificaciones afecta el comportamiento térmico del proyecto.

La teoría probabilística aporta el análisis de sensibilidad global como manera de evaluar la influencia de cada parámetro sobre el rango completo de variación de los resultados y el efecto de cada parámetro sobre los demás. Para los fines de este estudio, se propuso un modelo arquitectónico estándar y estadísticamente representativo del mercado de edificios de oficina en Santiago de Chile, simulado con el software de desempeño energético en régimen dinámico TAS. La FIGURA 1 presenta este modelo en planta -un piso tipo de 16 x 16 m y simétrico en los dos ejes- para el cual ocho recintos fueron analizados coincidentemente con las ocho orientaciones básicas. El clima utilizado para las simulaciones corresponde al de la ciudad de Santiago, el cual se puede caracterizar como templado cálido con estación seca prolongada de ocho a siete meses, de acuerdo a la clasificación de Köppen.

Al mismo tiempo, se seleccionaron cuatro parámetros de diseño para configurar el análisis de sensibilidad global. Estos parámetros fueron escogidos debido a que forman parte de las definiciones de diseño fundamentales del proyecto y probablemente tendrían influencia en el desempeño energético del futuro edificio. Luego, las variantes en cada uno de ellos representan el rango característico de decisiones a las que un arquitecto se enfrenta a la hora de diseñar un edificio de estas características de acuerdo a, por ejemplo, la oferta disponible en el mercado de soluciones de vidrio, muros cortina o protecciones solares. Estos cuatro parámetros serán estudiados en relación a su impacto sobre la demanda de enfriamiento del modelo simulado, en términos de $\mathrm{kWh} / \mathrm{m}^{2} / \mathrm{año.}$

Porcentaje de acristalamiento de la envolvente / Para las alternativas de 20\% -edificio en base a muros opacos y ventanas-, 50\% -solución mixta-y 100\% -edificio de muros cortina, completamente vidriadosde acristalamiento con respecto a la fachada completa. Este parámetro resulta particularmente interesante, por cuanto establece dos tipos de envolvente que podrían entenderse como opuestas y que además constituyen edificios formalmente muy diferentes entre sí.

Tipo de protección solar / Para las alternativas de alero en orientación Norte, más celosías en orientaciones Este y Oeste; versus solamente celosías en orientaciones Norte, Este y Oeste; y finalmente sin ningún tipo de protección solar. Este parámetro corresponde a uno de los elementos arquitectónicos probablemente más atractivos y más utilizados en la arquitectura del último tiempo para este tipo de edificios. Hoy en día, prácticamente existe consenso entre los arquitectos de la necesidad de incorporar estos elementos en la envolvente cuando se trata de un clima mediterráneo y especialmente si el edificio posee un alto porcentaje de acristalamiento. Las variantes de este parámetro recogen además las posibilidades de contar con aleros -recomendables para la orientación Norte- y/o de celosías -habitualmente asociadas a las orientaciones Oriente y Poniente- como protección solar.

1 Este texto se elabora en el marco del proyecto FONDECYT 1090602, "Enfriamiento ambiental de edificios de oficina a través de ventilación nocturna".

2 En este caso, el desempeño energético del proyecto estará asociado exclusivamente con las demandas de enfriamiento del modelo simulado. La demanda de enfriamiento se entenderá como la energía necesaria -en unidades de $\mathrm{kWh}$ - para mantener la temperatura interior de los recintos en el rango de confort deseado -para los fines de este estudio, se asume una temperatura máxima de $26^{\circ} \mathrm{C}$, de acuerdo al estándar EN15251:2007 (RHE/2)-. Este concepto se diferencia del de consumo de enfriamiento, que no considera el rendimiento de los sistemas mecánicos de refrigeración, por ejemplo el coP -Coefficient of Performance- de los equipos de aire acondicionado. La ventaja de la demanda por sobre el consumo, como indicador del desempeño energético, es que, al ser independiente del sistema de climatización utilizado, permite evaluar de mejor manera las modificaciones que se realicen en términos de diseño arquitectónico. 
FIG.01 Esquema de organización en planta del modelo arquitectónico utilizado para las simulaciones. Fuente: elaboración propia.

FIG.02 Coeficientes de correlación (r) con respecto a la demanda de enfriamiento obtenido para los cuatro parámetros de diseño estudiados. Fuente: elaboración propia.

FIG.03 Demanda de enfriamiento anual con respecto al porcentaje de acristalamiento en términos de frecuencia acu mulada. Fuente: elaboración propia.

Tipo de vidrio / Para las alternativas de vidrio simple claro, vidrio simple selectivo, vidriado doble claro y vidriado doble selectivo, que corresponden a los tipos de vidrio más utilizados actualmente dentro del mercado nacional. La diferencia entre estos estaría dada, entre otras propiedades, por la transmitancia térmica, donde el doble vidriado posee un valor más bajo y por ende pierde menos calor en comparación con el vidriado simple y por la transmitancia luminosa, en que el vidrio claro deja pasar aproximadamente un $90 \%$ de la luz visible, mientras que un vidrio selectivo lo puede llegar a hacer hasta en aproximadamente un $50 \%$, lo que limita las ganancias indeseadas de luz y calor y reduce, en teoría, el riesgo de sobrecalentamiento-.

Orientación / Para todas las orientaciones posibles: N, NE, E, SE, S, SW, W y NW. En este caso, el impacto de las distintas orientaciones sobre el comportamiento térmico de una edificación es de amplio conocimiento. Para un clima mediterráneo del hemisferio sur, es muy probable que el riesgo de sobrecalentamiento en la orientación Sur sea menor y que la orientación Poniente probablemente sea la más problemática desde el punto de vista del confort térmico de verano. La duda que queda es cuánto en verdad pesa esta variable con respecto a los otros parámetros de diseño en el modelo propuesto.

\section{ANÁLISIS DE SENSIBILIDAD}

Para este análisis de sensibilidad global, se consideró un total de 288 simulaciones, correspondientes a todas las posibles combinaciones de variantes por cada parámetro. El indicador utilizado para evaluar el nivel de importancia relativo de cada parámetro de diseño, con respecto a la demanda de enfriamiento, es el coeficiente de correlación de Pearson (r). Este coeficiente como valor definido, para este caso entre 0 y 1 , representa la medida del nivel en que los valores de
FIG.04 Demanda de enfriamiento anual con respecto al tipo de protección solar en términos de frecuencia acumulada. Fuente: elaboración propia

FIG.05 Demanda de enfriamiento anual con respecto al tipo de vidrio en términos de frecuencia acumulada. Fuente: ela boración propia.

FIG.06 Demanda de enfriamiento anual con respecto a la orientación en términos de frecuencia acumulada. Fuente: elaboración propia

(1)

FIG.01 >

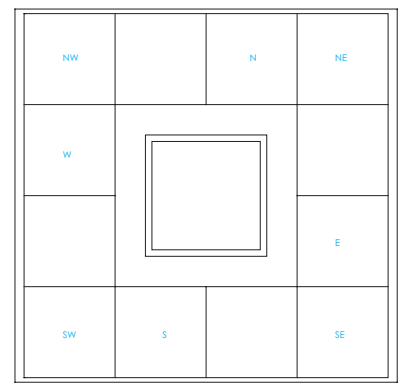

$\begin{array}{lllll}1 & 1 & 1 & 1 & 1 \\ 0 & 1 & & 4 m\end{array}$

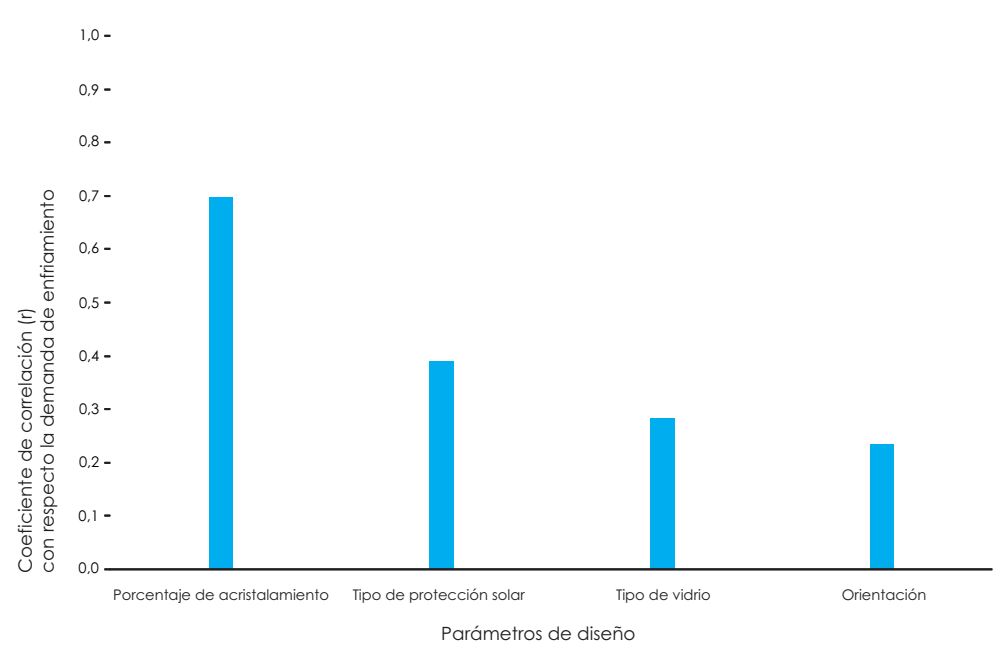

FIG.02 >

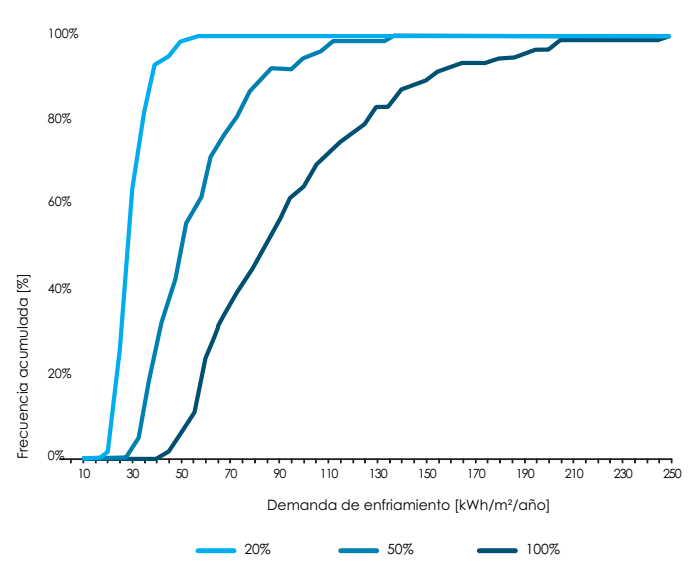

FIG.03 >

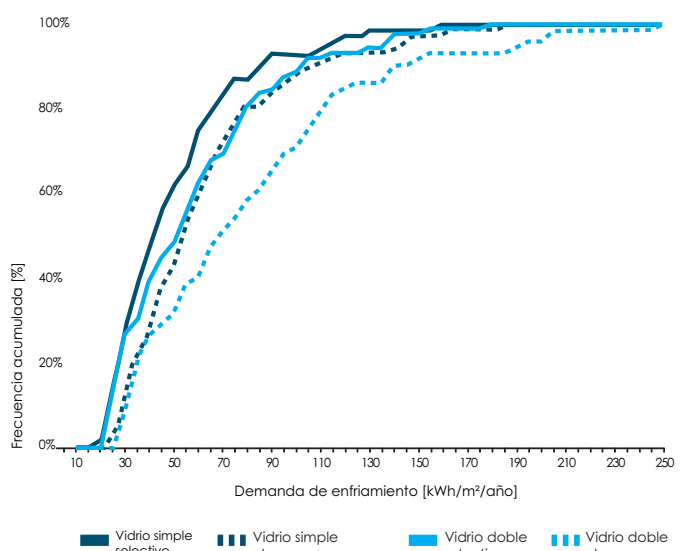

FIG.05 >
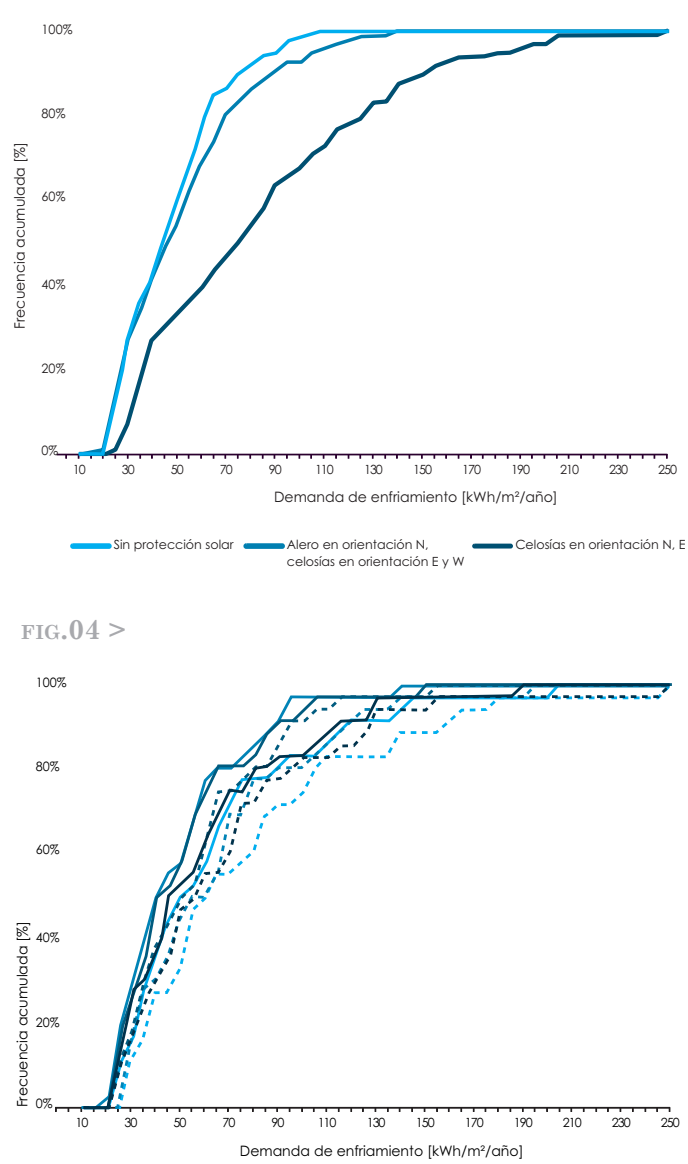

- ${ }^{n}$ - E $-s-W \quad$... NE ...SE ... SW ... NW

FIG.06 > 
los parámetros de entrada están linealmente correlacionados con los resultados. Mientras mayor sea el valor de r, más fuerte será la correlación lineal entre los datos de entrada y los datos de salida del modelo. Un valor de 1 significará entonces, una correlación perfecta, mientras que un valor de 0 significará que no existe una relación lineal entre las variables. Para los fines de este estudio, valores de $r$ menores a 0,30 se considerarán correlaciones débiles, mientras que sobre 0,70 serán entendidos como correlaciones fuertes.

La FIGURA 2 presenta los coeficientes de correlación obtenidos para los cuatro parámetros de diseño previamente descritos con respecto a la demanda de enfriamiento. De acuerdo a estos, es posible ordenar los distintos parámetros según el nivel de influencia que tengan sobre los resultados de las simulaciones. En consecuencia, mayores valores de r estarán asociados a variables más influyentes, desde el punto de vista de su impacto con respecto a la demanda de enfriamiento.

La correlación más fuerte está dada claramente por el porcentaje de acristalamiento $(r=0,70)$, por lo que se constituye como el parámetro más importante. En segundo lugar, aparece el tipo de protección solar, con un coeficiente de correlación moderado $(\mathrm{r}=0,39)$. Finalmente, con coeficientes de correlación bastante más bajos, aparecen el tipo de vidrio $(r=0,28)$ y la orientación $(r=0,23)$, que se convierten en las variables que menor influencia tienen sobre la demanda de enfriamiento. Hay que mencionar que, para todos estos casos, los coeficientes de correlación obtenidos poseen significancia estadística -para $p<0,05-$, lo que significa que la probabilidad de que estos resultados sean producto del azar es reducida -menor al 5\%-.

Estas conclusiones representan, a todas luces, observaciones relevantes en el contexto del diseño arquitectónico, puesto que orientan al arquitecto acerca de cuáles son las variables que debiese priorizar con miras a obtener un buen desempeño energético en su proyecto. Por ejemplo, si la elección del tipo de vidrio es una decisión importante, más aun lo será la elec- ción de la protección solar. Esto, en términos físicos, lo podemos entender por el hecho de que la protección solar, cuando es instalada por el exterior, posee suficientes elementos opacos y está cuidadosamente diseñada en función de la trayectoria solar, constituye la primera barrera contra el ingreso de radiación solar directa a los recintos, lo que reduce el riesgo de sobrecalentamiento. El desafío para el arquitecto será entonces poder combinar, de la manera más eficiente posible, la selección de la protección solar con el tipo de vidrio más adecuado.

Otra manera de entender el análisis de sensibilidad es a través de la observación directa de la variabilidad en los datos de salida de las simulaciones con respecto a cada uno de los parámetros de entrada. Las FIGURAS 3 a 6 nos presentan este tipo de análisis, para los parámetros de porcentaje de acristalamiento, tipo de protección solar, tipo de vidrio y orientaciones, respectivamente. Cada uno de estos gráficos está construido a partir de la frecuencia acumulada (de 0 a 100\%) con respecto a la demanda de enfriamiento.

La FIGURA 3, asociada al porcentaje de acristalamiento, y coincidentemente con el mayor valor $\mathrm{r}$ obtenido, es la que presenta el rango de variabilidad más amplio en relación a la demanda de enfriamiento entre todos los parámetros estudiados. Por ejemplo, el 99\% de los casos, correspondientes al $20 \%$ de acristalamiento, están asociados a una demanda de enfriamiento menor a $50 \mathrm{kWh} / \mathrm{m}^{2} / a n ̃ o$, que podría considerarse como baja. Por el contrario, en el caso de la envolvente completamente vidriada, solo un escaso $6 \%$ está por debajo de los $50 \mathrm{kWh} / \mathrm{m}^{2} /$ año y el resto se distribuye muy ampliamente, llegando incluso a valores muy altos de demanda de enfriamiento.

La FIGURA 6, correspondiente al parámetro de las orientaciones, muestra, por el contrario, un margen mucho más estrecho de variabilidad. Esto se puede observar gráficamente en el hecho que la distancia entre las curvas es mucho más reducida en comparación al caso anterior de la figura 3. Esto significa que la influencia de esta variable sobre los resultados de la demanda de enfriamiento es mucho menos relevante.
¿CÓMO DISEÑAR EDIFICIOS EN UN CLIMA MEDITERRÁNEO?

El ejercicio anteriormente descrito mostró cómo los cuatro parámetros de diseño propuestos impactan en distintos niveles el desempeño energético, durante el periodo de enfriamiento de un modelo estándar de edificio, en un clima mediterráneo como el de Santiago de Chile. Los resultados resultan sorprendentes en comparación con el supuesto inicialmente mencionado en relación a la orientación, puesto que contravienen la idea preconcebida de que esta sería una de las variables más relevantes -sino la más relevante- respecto del desempeño energético en refrigeración.

La elección del tipo de vidrio también presenta una situación interesante. En el grafico de la FIGURA 5 se puede observar cómo la diferencia entre tener vidrio claro y otro con una característica selectiva resulta mucho más relevante cuando se trata de doble vidriado. Esto se entiende por el hecho de que el vidriado doble puede ofrecer elevar las probabilidades de generación de sobrecalentamiento - por cuanto reduce la posibilidad de perder calor por la envolvente-, con lo que se hace necesario incorporar alguna propiedad al vidrio que ayude justamente a lo contrario, es decir, a reducir la ganancia solar, como es el caso del vidrio selectivo. Esta observación es importante, pues hoy en día el doble vidriado se ha instalado como un estándar generalizado de calidad constructiva de la envolvente en gran parte de los edificios de oficina que se construyen en Santiago de Chile. La recomendación en este caso, a la luz de los resultados, es claramente incorporar la propiedad selectiva como parte de la solución constructiva de los paramentos vidriados. La FIGURA 7 presenta una aplicación arquitectónica de un tipo de vidriado doble selectivo en la envolvente transparente de un edificio de oficinas en Santiago.

El gráfico de la FIGURA 4, en cambio, no muestra grandes diferencias entre las dos alternativas de protección solar estudiadas, pero para cualquiera de estos dos casos, sí aparece una brecha muy importante cuando se les 
compara con la variante sin ningún tipo de protección solar. Esta situación sugiere que la protección solar va a ser altamente aconsejable en la mayoría de los casos y prácticamente imprescindible cuando se trate de un edificio con envolvente $100 \%$ vidriada. $\mathrm{Al}$ respecto, la FIGURA 8 nos muestra una aplicación arquitectónica de protecciones solares de tipo celosías en paramentos vidriados de orientación Poniente en Santiago.

Finalmente, la gran brecha, en términos de demandas de enfriamiento, que aparece asociada al porcentaje de acristalamiento constituye una de las observaciones más importantes de este estudio. El desafío de diseño para los arquitectos que se abre a partir de esta situación es enorme. Aquí, más que nunca, las decisiones de diseño tienen un alto impacto en el que será el desempeño energético futuro del edificio. En la medida en que se pueda integrar los parámetros del tipo de protección solar y tipo de vidrio, junto con el diseño de la envolvente a partir de su porcentaje de acristalamiento, en una visión global del proyecto desde la perspectiva de su desempeño energético, podremos obtener edificaciones con mejores estándares de consumo de climatización y de confort térmico interior. De allí, la búsqueda de una transparencia adecuada, que combine elementos opacos con superficies vidriadas, será fundamental para conseguir este objetivo. La clave estará, por lo tanto, en un diseño arquitectónico informado, que integre todos los parámetros que afecten el desempeño energético del proyecto, tal como se hizo, desde una herramienta de análisis estadístico, en el estudio de sensibilidad global presentado en este artículo. +

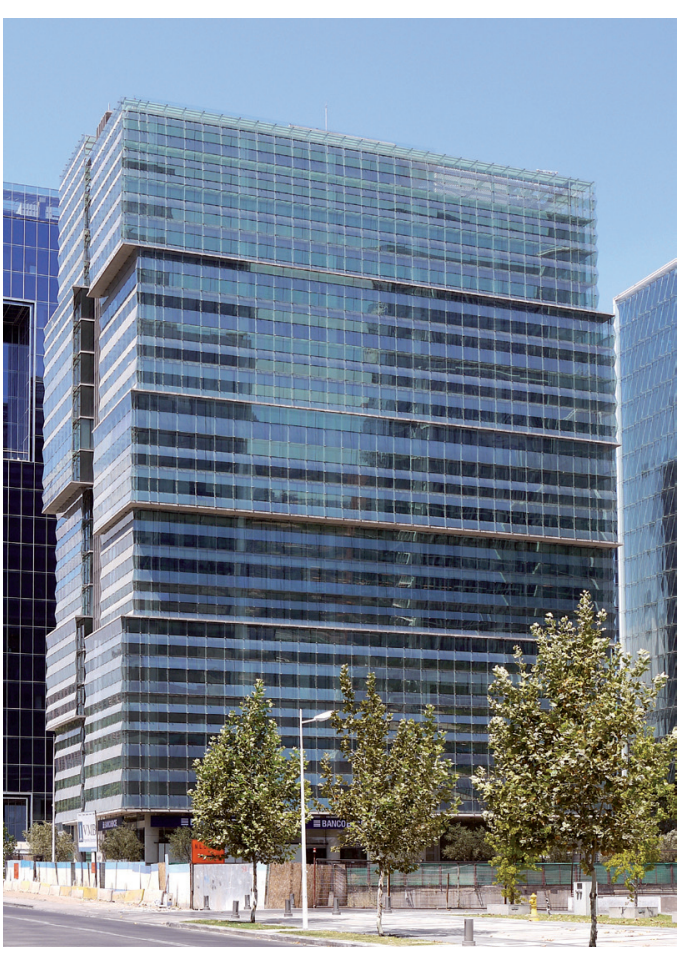

FIG.07 >

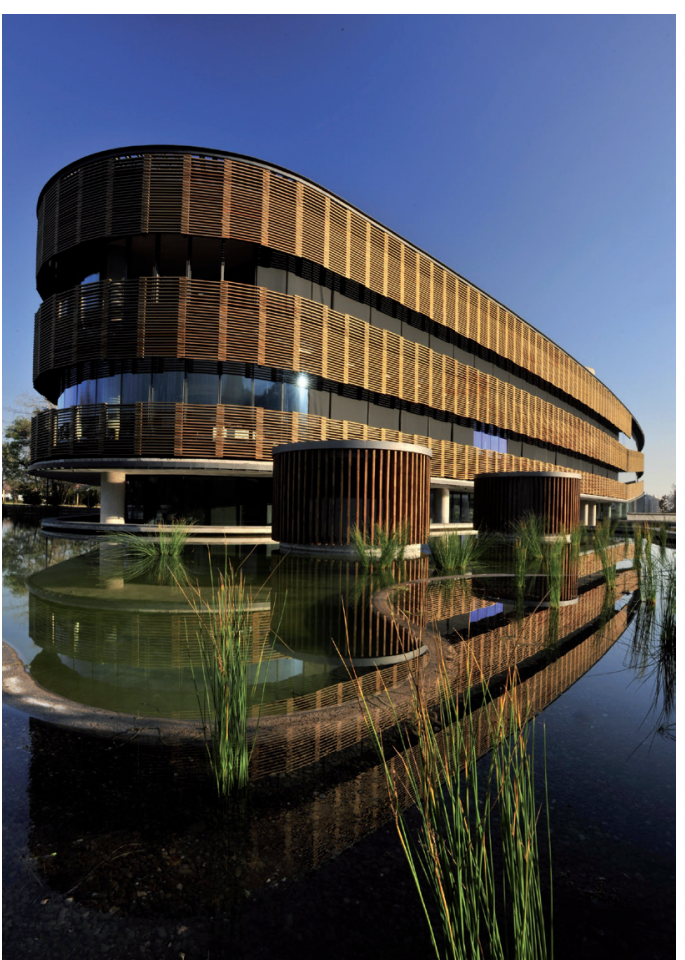

